\title{
Hadamard Multipliers and Abel Dual of Hardy Spaces
}

\author{
Pawel Mleczko \\ Faculty of Mathematics and Computer Science, Adam Mickiewicz University, Umultowska 87, 61-614 Poznań, Poland
}

Correspondence should be addressed to Paweł Mleczko; pml@amu.edu.pl

Received 18 February 2016; Revised 1 April 2016; Accepted 3 May 2016

Academic Editor: Antonio S. Granero

Copyright (C) 2016 Paweł Mleczko. This is an open access article distributed under the Creative Commons Attribution License, which permits unrestricted use, distribution, and reproduction in any medium, provided the original work is properly cited.

\begin{abstract}
The paper is devoted to the study of Hadamard multipliers of functions from the abstract Hardy classes generated by rearrangement invariant spaces. In particular the relation between the existence of such multiplier and the boundedness of the appropriate convolution operator on spaces of measurable functions is presented. As an application, the description of Hadamard multipliers into $H^{\infty}$ is given and the Abel type theorem for mentioned Hardy spaces is proved.
\end{abstract}

\section{Introduction}

In this paper we analyze so-called Hadamard multipliers between Hardy spaces of analytic functions. Our approach is rather general since we study Hardy spaces generated by rearrangement invariant spaces. The source of inspiration for the current research lies in the paper of Caveny [1] and a recent article of Blasco and Pavlović [2], where the Hadamard multipliers of classical Hardy spaces (see [1]) as well as related notions in more general settings (see [2]) were surveyed.

Let $H(\mathbb{D})$ denote the space of analytic function on the unit $\operatorname{disc} \mathbb{D}:=\{z \in \mathbb{C}:|z|<1\}$. Given spaces $A, B$ of sequences modelled on $\mathbb{N}_{0}:=\mathbb{N} \cup\{0\}$, an element $\left\{\lambda_{n}\right\}_{n \in \mathbb{N}_{0}} \subset \mathbb{C}$ is a multiplier of $A$ and $B$ if $\left\{\lambda_{n} \xi_{n}\right\}_{n \in \mathbb{N}_{0}} \in B$ for all $\left\{\xi_{n}\right\}_{n \in \mathbb{N}_{0}} \in A$. We study multipliers between spaces of analytic functions $E=$ $E(\mathbb{D}) \subset H(\mathbb{D})$, identifying with a space $E$ a space $\widehat{E}$ consisting of Taylor's coefficients of functions from $E$; that is,

$$
\widehat{E}=\left\{\left\{a_{n}\right\}: \sum_{n=0}^{\infty} a_{n} z^{n} \in E\right\} .
$$

It should be pointed out that in general the description of $\widehat{E}$ is a considerable challenging quest even for the classical spaces $E$, for example, Hardy spaces $H^{p}$ (see [3]). While it is clear that $\widehat{H^{2}}=\ell^{2}$, the characterization of $\widehat{H^{p}}$ is unknown if $p \neq 2$, $p<\infty$. As a matter of fact, there exists a nice description of $\widehat{H^{\infty}}$ in terms of convolution operator; see Schur's theorem in [4]. Note also the famous Hausdorff-Young inequality, which adjudicates that $\widehat{H^{p}} \subset \ell^{p^{\prime}}$ whenever $p \in[1,2]$ and
$1 / p+1 / p^{\prime}=1$. We also recall (after [5]) that when restricted to the nonnegative sequences $\left\{a_{n}\right\}$ satisfying $a_{n} \downarrow 0$, then

$$
\begin{aligned}
& \left\{\left\{a_{n}\right\}: \sum_{n=0}^{\infty} a_{n} z^{n} \in H^{p}\right\} \\
& \quad=\left\{\left\{a_{n}\right\}: \sum_{n=0}^{\infty}(n+1)^{p-2} a_{n}^{p}<\infty\right\}, \quad p \in(1, \infty) .
\end{aligned}
$$

Multipliers when considered between spaces of analytic functions are often called Hadamard multipliers (or Hadamard products); that is, given spaces of analytic functions $E$ and $F$ on $\mathbb{D}$ the Hadamard multipliers of $E$ and $F$ are defined as

$$
E \odot F=\left\{\sum_{n=0}^{\infty} a_{n} b_{n} u_{n}:\left\{a_{n}\right\} \in \widehat{E},\left\{b_{n}\right\} \in \widehat{F}\right\},
$$

where $u_{n}: \mathbb{D} \rightarrow \mathbb{C}$ is given by $u_{n}(z)=z^{n}, z \in \mathbb{D}$, $n \in \mathbb{N}_{0}$. We refer the reader to $[2,6]$ for more information and background on this topic.

Motivated by the mentioned paper of Caveny [1] we study the Hadamard product between abstract Hardy spaces. Note that in [1] Hadamard multipliers were considered within the settings of Hardy spaces $H^{p}, p \in[1, \infty]$. We point out that the study of Hadamard product is a well established issue in the theory of spaces of analytic functions (see, e.g., [6]); however, most studies have concentrated on the classical case of Hardy spaces $H^{p}$. The purpose of this paper is to extend the research to more general case of Hardy spaces generated by r.i. spaces. 


\section{Rearrangement Invariant and Abstract Hardy Spaces}

Let $(\Omega, \Sigma, \mu)$ be a complete $\sigma$-finite measure space and let $L^{0}(\Omega):=L^{0}(\Omega, \Sigma, \mu)$ denote the space of real valued measurable functions on $\Omega$ with the topology of convergence in measure on $\mu$-finite sets. The order $|x| \leqslant|y|$ means that $|x(\omega)| \leqslant|y(\omega)|$ for $\mu$-almost all $\omega \in \Omega$. If a real Banach space $X \subset L^{0}(\Omega)$ is such that there exists $u \in X$ with $u>0 \mu$-a.e. on $\Omega$ and $|x| \leqslant|y|$ with $x \in L^{0}(\Omega)$ and $y \in X$ implies $x \in X$ with $\|x\|_{X} \leqslant\|y\|_{X}$, then $X$ is said to be a Banach lattice (on $\Omega$ or on $(\Omega, \mu)$ ). If in addition, $X$ contains, along with a function $f$, every function $g$ equimeasurable with $f,\|f\|_{X}=\|g\|_{X}$, then we say that $X$ is rearrangement invariant (r.i. space for short).

Throughout the paper we will consider complex r.i. spaces. The term complex r.i. space refers to the complexification of a real r.i. space; that is, if $X$ denotes the (real) r.i. space, the complexification $X(\mathbb{C})$ of $X$ is the Banach space of all complex valued measurable functions $x$ on $\Omega$ such that the element $|x|$ defined by $|x|(\omega)=|x(\omega)|$ for $\omega \in \Omega$ is in $X$ and $\|x\|=$ $\||x|\|_{X}$. For the simplicity of presentation, we will often write r.i. space instead of a complex r.i. space and avoid the use of symbol $X(\mathbb{C})$. An (real) r.i. function space $X$ on $(\Omega, \mu)$ is said to be order continuous if every nonnegative nonincreasing sequence in $X$ which converges a.e. to 0 converges to 0 in the norm topology of $X$. If $X$ is an order continuous r.i. space on $(\Omega, \Sigma, \mu)$, then the dual space $X^{*}$ can be identified in a natural way with the Köthe dual space $\left(X^{\prime},\|\cdot\|_{X^{\prime}}\right)$ of all $x \in L^{0}(\Omega)$ such that $x y \in L^{1}(\Omega)$, for all $y \in X$. An r.i. space $X$ is said to be maximal (or to have the Fatou property), if, for any sequence $\left(x_{n}\right)$ of nonnegative elements from $X$ such that $x_{n} \uparrow x$ for $x \in L^{0}(\Omega)$ and $\sup \left\{\left\|x_{n}\right\|_{X}: n \in \mathbb{N}\right\}<\infty$, one has $x \in X$ and $\left\|x_{n}\right\|_{X} \rightarrow\|x\|_{X}$.

In the sequel we will use the well-known concept of Boyd indices. Recall that for an r.i. space $X$ on $\mathbb{T}$ we define dilation operators $D_{s}: X \rightarrow X, s>0$, by $D_{s} f(t)=f(t / s)$ for all $t \leqslant 2 \pi \min \{1, s\}$ and $D_{s} f(t)=0$ for $2 \pi s<t \leqslant 2 \pi$. Boyd indices $p_{X}$ and $q_{X}$ are then defined by

$$
\begin{aligned}
& p_{X}=\lim _{s \rightarrow \infty} \frac{\log s}{\log \left\|D_{s}\right\|}, \\
& q_{X}=\lim _{s \rightarrow 0^{+}} \frac{\log s}{\log \left\|D_{s}\right\|} .
\end{aligned}
$$

It follows that $p_{X}, q_{X} \in[1, \infty]$ and $p_{X} \leqslant q_{X}$. Moreover $1 / p_{X}+$ $1 / p_{X^{\prime}}=1$ and $1 / q_{X}+1 / q_{X^{\prime}}=1$ (see $[7$, p. 131]).

Let $X$ be a complex r.i. space on $\mathbb{T}:=[0,2 \pi)$. Denote by $H X$ the space of all $f \in H(\mathbb{D})$ such that $\sup \left\{\left\|f_{r}\left(e^{i \cdot}\right)\right\|_{X}: r \in\right.$ $[0,1)\}<\infty$, where $f_{r}: \partial \mathbb{D} \rightarrow \mathbb{C}$ is given by $f_{r}\left(e^{i \theta}\right)=f\left(r e^{i \theta}\right)$, $\theta \in \mathbb{T}$. The space $H X$ becomes a Banach space if equipped with the norm

$$
\|f\|_{H X}:=\sup \left\{\left\|f_{r}\left(e^{i \cdot}\right)\right\|_{X}: r \in[0,1)\right\} .
$$

Note that the spaces $H X$ are abstract variants of the classical Hardy spaces $H^{p}$ on the disc, $p \in[1, \infty]$ (see [3]), as well as other important spaces of analytic functions like HardyOrlicz, Hardy-Lorentz, and Hardy-Marcinkiewicz spaces; see, for example, [8-10] for the recent studies on this topic.
The study of functions analytic on the disc is intimately connected to the study of the boundary functions. Recall that the radial limit $f^{*}$ of $f \in H(\mathbb{D})$ is given by $f^{*}\left(e^{i \theta}\right)=$ $\lim _{r \rightarrow 1^{-}} f_{r}\left(e^{i \theta}\right)$ provided that the limit exists for almost all $\theta \epsilon$ $\mathbb{T}$. By the lemma of Fatou it follows that if $f=\sum_{n=0}^{\infty} \widehat{f}(n) u_{n} \epsilon$ $H X$, then $f^{*}$ exists a.e. on $\mathbb{T}$. Here and beneath, for a function $f \in H(\mathbb{D})$, we will write $\hat{f}(n)$ for $n$th Taylor's coefficient of $f$, $n \in \mathbb{N}_{0}$. It is clear that the series $\sum_{n=0}^{\infty} \widehat{f}(n) u_{n}$ is convergent uniformly on compact subsets of $\mathbb{D}$ to the function $f$. For an r.i. space $X$ we use the symbol $\widetilde{H X}$ to denote the subspace of elements from $X$ consisting of radial limits of $H X$ functions. It was shown in [8, Proposition 2.2] that if $X$ is a maximal r.i. space on $\mathbb{T}$ then $H X$ coincides with the space consisting of functions $f \in X$ such that the negative Fourier coefficients of $f$ vanish. The mapping $f \mapsto f^{*}$ appeared to be an isometric isomorphism from $H X$ into $X$.

The key fact in [1] for the study of Hadamard product of $H^{p}$ spaces was the so-called Riesz property. Let $R$ be a linear projection given by

$$
R\left(\sum_{k=-n}^{n} a_{k} e^{k i t}\right)=\sum_{k=0}^{n} a_{k} e^{k i t}, \quad t \in \mathbb{T}, n \in \mathbb{N}_{0} .
$$

It was Riesz who proved that $R$ extends to the bounded projection on $L^{p}(\mathbb{T})$ if only $p \in(1, \infty)$ (see, e.g., [7]). Let $X$ be an r.i. space on $\mathbb{T}$. We say that $X$ has the Riesz property if, for every $f \in X$ with the Fourier coefficients $\{\hat{f}(n)\}$, the function $\sum_{n=0}^{\infty} \hat{f}(n) u_{n}$ belongs to $H X$ and there exists a constant $C>0$ such that

$$
\left\|\sum_{n=0}^{\infty} \widehat{f}(n) u_{n}\right\|_{H X} \leqslant C\|f\|_{X} .
$$

It is easy to see that an r.i. space $X$ has the Riesz property if its Boyd indices $p_{X}, q_{X}$ satisfy $p_{X}, q_{X} \in(1, \infty)$. In fact, much more can be proved. In [11] it was shown that $X$ has the Riesz property if and only if $p_{X}, q_{X} \in(1, \infty)$. In particular, it follows that an r.i. space $X$ has the Riesz property if and only if $X^{\prime}$ does.

\section{Hadamard Multipliers}

We study the Hadamard product of functions from Hardy classes generated by r.i. spaces. Given functions $f, g \in H(\mathbb{D})$ the Hadamard product of $f$ and $g$ is given by the formula

$$
f \odot g=\sum_{n=0}^{\infty} \widehat{f}(n) \hat{g}(n) u_{n} .
$$

We note that by the Cauchy-Hadamard formula $f, g \in H(\mathbb{D})$ implies that $f \odot g \in H(\mathbb{D})$.

Let $H X$ and $H Y$ be abstract Hardy spaces generated by r.i. spaces $X$ and $Y$, respectively. It is easy to verify that the functional given by

$$
\|g\|_{H X \odot H Y}=\sup \left\{\|f \odot g\|_{H Y}:\|f\|_{H X} \leqslant 1\right\}
$$

is a norm on $H X \odot H Y$ and we have a continuous inclusion $H X \odot H Y \hookrightarrow H(\mathbb{D})$. Combining this with the Closed Graph 
Theorem easily implies that $H X \odot H Y$ is a Banach space equipped with the norm given in (9).

Before we state and prove a technical lemma which will be useful in the sequel we recall that the space $L^{1}=L^{1}(\partial \mathbb{D})$ is a Banach algebra under the convolution $*$ given by

$$
\begin{aligned}
(f * g)\left(e^{i \theta}\right)=\frac{1}{2 \pi} \int_{0}^{2 \pi} f\left(e^{i t}\right) g\left(e^{i(\theta-t)}\right) d t, & \\
& f, g \in L^{1}, \theta \in \mathbb{T} .
\end{aligned}
$$

The function $f * g$ which is measurable by Fubini's Theorem belongs to $L^{1}(\partial \mathbb{D})$ and satisfies

$$
\|f * g\|_{L^{1}} \leqslant\|f\|_{L^{1}}\|g\|_{L^{1}} .
$$

Lemma 1. Let $f, g \in H(\mathbb{D})$ and let $X$ be an r.i. space on $\mathbb{T}$. Then the following hold:

(i) $(f \odot g)_{r}=f_{r} * g_{r}$ for every $r \in[0,1)$.

(ii) If $f \in H X$, then $(f \odot g)_{r}=f^{*} * g_{r}$ for every $r \in[0,1)$.

Proof. (i) Given any $r \in[0,1)$ and $\theta \in \mathbb{T}$ the series

$$
\sum_{n=0}^{\infty} \widehat{g}(k) r^{k} e^{i n(\theta-t)}, \quad t \in \mathbb{T}
$$

converges uniformly on $\mathbb{T}$. Thus the sequence

$$
\left\{\sum_{n=0}^{\infty} \widehat{\mathcal{g}}(k) r^{k} e^{i n(\theta-t)}\right\}_{n \in \mathbb{N}_{0}}
$$

is bounded on $\mathbb{T}$. Since $f_{r}$ is a bounded function on $\partial \mathbb{D}$ we have

$$
\begin{aligned}
& \left(f_{r} * g_{r}\right)\left(e^{i \theta}\right) \\
& \quad=\frac{1}{2 \pi} \int_{0}^{2 \pi} f\left(r e^{i t}\right)\left(\sum_{n=0}^{\infty} \widehat{g}(n) r^{n} e^{i n(\theta-t)}\right) d t \\
& \quad=\sum_{n=0}^{\infty} \widehat{g}(n) r^{n} e^{i n \theta}\left(\int_{0}^{2 \pi} f\left(r e^{i t}\right) e^{-i n t} d t\right) \\
& \quad=\sum_{n=0}^{\infty} \widehat{f}(n) \hat{g}(n)\left(r e^{i \theta}\right)^{n}=(f \odot g)\left(r e^{i \theta}\right) .
\end{aligned}
$$

(ii) Let $f \in H X$. From [9, Proposition 2.2] it follows (by $\left.X^{\prime \prime} \subset L^{1}(\mathbb{T})\right)$ that $f^{*} \in L^{1}(\partial \mathbb{D})$ and Fourier coefficients of $f^{*}$ satisfy for $n<0$

$$
\widehat{f^{*}}(n)=\frac{1}{2 \pi} \int_{0}^{2 \pi} f^{*}\left(e^{i t}\right) e^{-i n t} d t=0
$$

and for $n \in \mathbb{N}_{0}$

$$
\widehat{f^{*}}(n)=\widehat{f}(n)
$$

Combining the above proof of (i) with the Lebesgue Domination Theorem yields the second formulae.
In what follows $A(\mathbb{D})$ denotes the disc algebra, that is, the space of functions $f \in H(\mathbb{D})$ such that $f$ extends continuously to the closure $\overline{\mathbb{D}}$ of $\mathbb{D}$.

Corollary 2. Let $X, Y$, and $Z$ be r.i. spaces on $\mathbb{T}$. Then the inclusion

$$
H Z \subset H X \odot H Y
$$

holds if and only if the restriction of the convolution operator to $A(\mathbb{D}) \times A(\mathbb{D})$ is bounded from $X \times Z$ to $Y$; that is, there exists $C>0$ such that $\|f * g\|_{Y} \leqslant C\|f\|_{X}\|g\|_{Z}$ for all $f, g \in A(\mathbb{D})$.

Proof.

Sufficiency. By the Closed Graph Theorem we conclude that there exists $C>0$ such that $\|g\|_{H X \odot H Y} \leqslant C\|g\|_{H Z}$ for every $g \in H Z$. This implies that

$$
\|f \odot g\|_{H Y} \leqslant\|f\|_{H X}\|g\|_{H Z}, \quad f \in H X, g \in H Z
$$

and so, applying Lemma 1, we obtain the required statement on boundedness of the restriction of the convolution operator from $X \times Z$ to $Y$.

Necessity. Fix $g \in H Z$. Combining Lemma 1 with the boundedness of the convolution operator yields that there exists $C>0$ such that for all $f \in H X$ and all $r \in[0,1)$ we have

$$
\begin{aligned}
\left\|(f \odot g)_{r}\right\|_{Y} & =\left\|f_{r} * g_{r}\right\|_{Y} \leqslant C\left\|f_{r}\right\|_{X}\left\|g_{r}\right\|_{Z} \\
& \leqslant C\|f\|_{H X}\|g\|_{H Z} .
\end{aligned}
$$

Hence

$$
\|f \odot g\|_{H Y} \leqslant C\|f\|_{H X}\|g\|_{H Z}
$$

and so the continuous inclusion $H Z \hookrightarrow H X \odot H Y$ follows.

Here comes the main theorem of this section.

Theorem 3. Let $X, Y$, and $Z$ be maximal r.i. spaces on $\mathbb{T}$. Assume that $X$ or $Y$ is separable. Then the inclusion

$$
H Z \subset H X \odot H Y
$$

holds if and only if the convolution operator is bounded from $\widetilde{H X} \times \widetilde{H Y}$ to $Z$.

Proof. For any $f \in H X$ and $g \in H X \odot H Y$ we have

$$
\begin{aligned}
\left\|(f \odot g)_{r}\right\|_{Y} \leqslant\|f \odot g\|_{H Y} \leqslant\|f\|_{H X}\|g\|_{H X \odot H Y}, & \\
r & \quad(0,1) .
\end{aligned}
$$

Assume that $H Z \subset H X \odot H Y$. Then the Closed Graph Theorem implies that there exists $C$ such that $\|g\|_{H X \odot H Y} \leqslant$ $C\|g\|_{H Z}$ for every $g \in H Z$. Combining the above we conclude that for all $f \in H X$ and $g \in H Z$

$$
\left\|(f \odot g)_{r}\right\|_{Y} \leqslant C\|f\|_{H X}\|g\|_{H Z}, \quad r \in[0,1) .
$$


Since $\|f\|_{H X}=\|f\|_{\overparen{H X}}$ and $\|g\|_{H Z}=\|g\|_{\overparen{H Z}}$ by Lemma 1(ii) we obtain

$$
\left\|f^{*} * g_{r}\right\|_{Y} \leqslant C\|f\|_{\overparen{H X}}\|g\|_{\overparen{H Z}}, \quad r \in[0,1) .
$$

We will use the Mean Convergence Theorem from [9, Theorem 3.2] from which it follows (note that $Z$ is maximal, by assumption) that for every $g \in H Z$

$$
\lim _{r \rightarrow 1^{-}}\left\|g_{r}-g^{*}\right\|_{Z}=0 .
$$

Take any sequence $\left(r_{n}\right) \subset[0,1)$ such that $r_{n} \rightarrow 1$. Then (25) implies that there exist $G \in Z$ and a subsequence $\left(r_{k_{n}}\right)$ of $\left(r_{n}\right)$ such that

$$
\begin{gathered}
g_{r_{k_{n}}} \longrightarrow g^{*} \text { a.e. on } \mathbb{T} \text {, } \\
\left|g_{r_{k_{n}}}\right| \leqslant G \quad \text { a.e. on } \mathbb{T} \text {. }
\end{gathered}
$$

Now observe that for almost all $\theta, t \in \mathbb{T}$

$$
f^{*}\left(e^{i t}\right) g_{r_{k_{n}}}\left(e^{i(\theta-t)}\right) \longrightarrow f^{*}\left(e^{i t}\right) g^{*}\left(e^{i(\theta-t)}\right) .
$$

Since $f^{*} \in L^{1}(\partial \mathbb{D})$ and $G \in L^{1}(\partial \mathbb{D})$ it follows that

$$
\frac{1}{2 \pi} \int_{0}^{2 \pi}\left|f^{*}\left(e^{i t}\right) G\left(e^{i(\theta-t)}\right)\right| d t<\infty \quad \text { a.e. on } \mathbb{T}
$$

and so inequality (27) in combination with the Lebesgue Domination Theorem yields

$$
f^{*} * g_{r_{k_{n}}} \longrightarrow f^{*} * g^{*} \text { a.e. on } \mathbb{T} \text {. }
$$

The Fatou property of $Y$ and inequality (24) imply

$$
\begin{aligned}
\left\|f^{*} * g^{*}\right\|_{Y} & \leqslant \liminf _{n \rightarrow \infty}\left\|f^{*} * g_{r_{k_{n}}}\right\|_{Y} \leqslant C\left\|f^{*}\right\|_{X}\left\|g^{*}\right\|_{Z} \\
& =C\|f\|_{\overparen{H X}}\|g\|_{\overparen{H Y}}
\end{aligned}
$$

and this gives the assertion. The reverse implication follows from Corollary 2.

The following result gives a description of the Hadamard multipliers from Hardy spaces $H X$ into $H^{\infty}$ under some mild assumption on an r.i. space $X$.

Theorem 4. Let $X$ be a maximal r.i. space on $\mathbb{T}$ with the Boyd indices satisfying $p_{X}, q_{X} \in(1, \infty)$. Then

$$
H X \odot H^{\infty}=H X^{\prime} .
$$

Proof. Since the convolution operator is bounded from $X \times X^{\prime}$ into $L^{\infty}$ with

$$
\|f * g\|_{L^{\infty}} \leqslant\|f\|_{X}\|g\|_{X^{\prime}}, \quad(f, g) \in X \times X^{\prime}
$$

it follows from Corollary 2 that

$$
H X^{\prime} \hookrightarrow H X \odot H^{\infty} .
$$

To prove the reverse continuous inclusion we observe that our hypothesis on the Boyd indices implies that there exists a Riesz projection $\mathscr{R}: X \rightarrow \widetilde{H X}$ (see [11]). Thus there exists a constant $C=C_{X} \geqslant 0$ such that

$$
\|\mathscr{R} F\|_{\overparen{H X}} \leqslant C\|F\|_{X}, \quad F \in X .
$$

Let $g \in H X \odot H^{\infty}$. We claim that $g \in H X^{\prime}$. To see this note that $X^{\prime}$ is a maximal r.i. space and in consequence translation invariant. Since $L^{\infty}(\mathbb{T}) \subset X^{\prime}$ it follows that for every $r \in[0,1)$ and $\theta \in \mathbb{T}$

$$
\begin{aligned}
& \left\|g_{r}\right\|_{X^{\prime}} \\
& \quad=\sup \left\{\frac{1}{2 \pi}\left|\int_{0}^{2 \pi} F\left(e^{i t}\right) g_{r}\left(e^{i(\theta-t)}\right) d t\right|:\|F\|_{X} \leqslant 1\right\} .
\end{aligned}
$$

Combining inequality (35) with $\mathscr{R} X=\widetilde{H X}$ and $\|f\|_{H X}=$ $\left\|f^{*}\right\|_{X}$ for all $f \in H X$, we conclude that for every $\theta \in \mathbb{T}$

$$
\begin{aligned}
& \left\|g_{r}\right\|_{X^{\prime}} \leqslant C \\
& \cdot \sup \left\{\frac{1}{2 \pi}\left|\int_{0}^{2 \pi}(\mathscr{R} F)\left(e^{i t}\right) g_{r}\left(e^{i(\theta-t)}\right) d t\right|:\|\mathscr{R} F\|_{X}\right. \\
& \leqslant 1\}=C \\
& \cdot \sup \left\{\frac{1}{2 \pi}\left|\int_{0}^{2 \pi} f^{*}\left(e^{i t}\right) g_{r}\left(e^{i(\theta-t)}\right) d t\right|:\|f\|_{H X}\right. \\
& \leqslant 1\}=C \sup \left\{\left|\left(f^{*} * g_{r}\right)\left(e^{i \theta}\right)\right|:\|f\|_{H X} \leqslant 1\right\} .
\end{aligned}
$$

This implies by Lemma 1(ii) that

$$
\begin{aligned}
& \left\|g_{r}\right\|_{X^{\prime}} \\
& \quad \leqslant C \sup \left\{\left|\left(f^{*} g_{r}\right)\left(e^{i \theta}\right)\right|:\|f\|_{H X} \leqslant 1, r \in[0,1)\right\} \\
& \quad \leqslant C \sup \left\{\|f \odot g\|_{H^{\infty}}:\|f\|_{H X} \leqslant 1\right\} .
\end{aligned}
$$

This proves the claim that $g \in H X^{\prime}$ and so

$$
H X \odot H^{\infty} \hookrightarrow H X^{\prime}
$$

with continuous inclusion.

\section{Applications}

In the following we apply the former theorem to the study of the Abel duals of Hardy spaces. Recall that the Abel dual of a space $X \subset X(\mathbb{D})$ consists of all $g \in H(\mathbb{D})$ such that the limit

$$
\lim _{r \rightarrow 1^{-}} \sum_{n=0}^{\infty} \hat{f}(n) \hat{g}(n) r^{n}
$$

exists for all $f \in X$ (see [12, p. 1223]). We prove the version of the identification of the Abel dual of abstract Hardy spaces in the following. 
Theorem 5. Let $X$ be an order continuous and maximal ri. space on $\mathbb{T}$ and assume that $X$ has the Riesz property. The following statements are equivalent.

(i) If the function $g \in H(\mathbb{D})$ can be represented in the form

$$
g=\sum_{n=0}^{\infty} \hat{g}(n) u_{n}
$$

then $f \odot g \in H^{\infty}$ for every $f \in H X$.

(ii) For any sequence of complex numbers $\left\{\lambda_{n}\right\}_{n \in \mathbb{N}_{0}}$ and any $f \in H X$, the following limit exists a.e. on $\mathbb{T}$ :

$$
\lim _{r \rightarrow 1^{-}} \sum_{n=0}^{\infty} \widehat{f}(n) \lambda_{n} r^{n} e^{i n \theta}
$$

Proof. (i) $\Rightarrow$ (ii). From Theorem 4 it follows that $g \in H X^{\prime}$ and since $X^{\prime}$ has the Riesz property, $g$ is a projection of some $G \epsilon$ $X^{\prime}$. Observe that for $0 \leqslant r<\rho<1$ and $0 \leqslant s<\rho<1$ the following equality holds:

$$
\begin{aligned}
& \int_{0}^{2 \pi} f(t)\left[g_{t}\left(e^{i(\theta-t)}\right)-g_{s}\left(e^{i(\theta-t)}\right)\right] d t \\
& \quad=\int_{0}^{2 \pi}\left[f_{r / \rho}\left(e^{i t}\right)-f_{s / \rho}\left(e^{i t}\right)\right] g_{\rho}\left(e^{i(\theta-t)}\right) d t .
\end{aligned}
$$

Hölder inequality yields

$$
\begin{aligned}
\mid f & \odot g\left(r e^{i \theta}\right)-f \odot g\left(s e^{i \theta}\right) \mid \\
& =\left|\frac{1}{2 \pi} \int_{0}^{2 \pi}\left[f_{r / \rho}(t)-f_{s / \rho}(t)\right] g_{s}(\theta-t) d t\right| \\
& \leqslant\left\|f_{r / \rho}-f_{s / \rho}\right\|_{X}\left\|g_{s}\right\|_{X^{\prime}} .
\end{aligned}
$$

Since $X$ is maximal, then from the Mean Convergence Theorem (see [9, Theorem 3.2]) it follows that

$$
\lim _{r \rightarrow 1^{-}} \sum_{n=0}^{\infty} \hat{f}(n) \lambda_{n} r^{n} e^{i n \theta}=\lim _{r \rightarrow 1^{-}} f \odot g\left(r e^{i \theta}\right) \quad \text { a.e. on } \mathbb{T} \text {. }
$$

(ii) $\Rightarrow\left(\right.$ i). Take $0<\delta<1$ and consider a function $f_{\delta}:=$ $\sum_{n=0}^{\infty} \delta^{n} u_{n}$. Since $L^{\infty}(\mathbb{T}) \subset X, f_{\delta} \in H X$. From (ii) it follows that

$$
\lim _{r \rightarrow 1^{-}} \sum_{n=0}^{\infty} \delta^{n} \lambda_{n} r^{n} e^{i n \theta}
$$

exists for almost all $\theta \in \mathbb{T}$ and hence

$$
\sum_{n=0}^{\infty} \lambda_{n} r^{n} e^{i n \theta}
$$

converges for $r \in[0,1)$. Thus

$$
g:=\sum_{n=0}^{\infty} \lambda_{n} u_{n} \in H(\mathbb{D}) .
$$

Take arbitrary $f \in H X$ and notice that by (ii)

$$
\begin{gathered}
\lim _{r \rightarrow 1^{-}} \frac{1}{2 \pi} \int_{0}^{2 \pi} f^{*}\left(e^{i t}\right) g_{r}\left(e^{i(\theta-t)}\right) d t \\
=\lim _{r \rightarrow 1^{-}}(f \odot g)\left(r e^{i \theta}\right)
\end{gathered}
$$

exists a.e. on $\mathbb{T}$. By the principle of uniform boundedness applied to the family $\left(f * g_{r}\right)_{r \in[0,1)}$ of operators $f \mapsto f * g_{r}$ on $H X$, it follows that there exists a constant $C>0$ such that

$$
\left|\frac{1}{2 \pi} \int_{0}^{2 \pi} f\left(e^{i t}\right) g_{r}\left(e^{i(\theta-t)}\right) d t\right| \leqslant C\|f\|_{H X}, \quad f \in H X
$$

and hence $f \odot g \in H^{\infty}$.

Further the description of the dual space of $H X$ will be needed. Recall that if $X$ is maximal r.i. spaces, then $H X=\widetilde{H X}$ with equality of norms and thus $\widetilde{H X}$ is a closed subspace of $X$. As in the proof for the case $X=L^{p}, p \in[1, \infty)$, to represent the dual of $(H X)^{*}$, it is sufficient to identify the annihilator of $\widetilde{H X}$ in $X^{*}$. The proof of the forthcoming lemma is standard and follows the steps of the proof of [3, Theorem 7.1]; nonetheless we include it here for the sake of completeness. The symbol $H X_{0}$ stands for the set of all $f \in H X$ such that $f(0)=0$.

Lemma 6. Let $X$ be an order continuous and maximal r.i. space on $\mathbb{T}$. Then the dual $X^{*}$ is isometrically isomorphic to $X^{\prime} / \widetilde{H X_{0}^{\prime}}$. If in addition $X$ has the Riesz property, then, for all $\varphi \in(H X)^{*}$, there exists a unique function $g \in \widetilde{H X^{\prime}}$ such that

$$
\varphi(f)=\frac{1}{2 \pi} \int_{0}^{2 \pi} f^{*}\left(e^{i \theta}\right) \overline{g^{*}\left(e^{i \theta}\right)} d \theta, \quad f \in H X .
$$

Proof. Assume that $X$ is an order continuous and maximal ri. space on $\mathbb{T}$. Every linear and bounded functional $\varphi$ on $X$ can be represented in the form

$$
\varphi(f)=\frac{1}{2 \pi} \int_{0}^{2 \pi} f\left(e^{i \theta}\right) g\left(e^{i \theta}\right) d \theta
$$

for some $g \in X^{\prime}$ such that $\|\varphi\|_{X^{*}}=\|g\|_{X^{\prime}}$. We will describe the annihilator of $\widetilde{H X}$ in $X^{\prime}$. Take $g \in X^{\prime}$ such that

$$
\int_{0}^{2 \pi} g\left(e^{i \theta}\right) f^{*}\left(e^{i \theta}\right) d \theta=0 \quad \forall f \in H X .
$$

Since polynomials are dense in $H X$, it follows that $\widehat{g}(n)=$ $\int_{\mathbb{T}} g\left(e^{i \theta}\right) e^{i n \theta} d \theta=0$ for all $n \in \mathbb{N}_{0}$ and then $g \in H X^{\prime}$ and $g(0)=0$. Now, by the assumption $X^{\prime}$ is maximal and thus we have $g \in H X_{0}^{\prime}$.

Conversely, assume that $g \in \widetilde{H X_{0}^{\prime}}$. Then by the Cauchy formulae it follows that $\int_{\mathbb{T}} f^{*}\left(e^{i \theta}\right) g\left(e^{i \theta}\right) d \theta=0$ for every $f \epsilon$ $H X$. Hence $\widetilde{H X_{0}}$ is the annihilator of $\widetilde{H X}$ and it follows that $(H X)^{*} \cong X^{\prime} / \widetilde{H X_{0}^{\prime}}$ isometrically.

Take $\varphi \in(H X)^{*}$ and observe that by the Hahn-Banach theorem $\varphi$ extends to a functional on $X$ and hence $\varphi$ can be 
represented in form (52) by a function $g \in X^{\prime}$. This extension is not unique; however, it becomes so if we distinguish in each coset a function $g$ for which $\widehat{g}(n)=0, n \in \mathbb{N}$. In other words

$$
\varphi(f)=\int_{0}^{2 \pi} f^{*}\left(e^{i \theta}\right) \overline{g\left(e^{i \theta}\right)} d \theta, \quad f \in H X .
$$

Note that $X^{\prime}$ has the Riesz property since $X$ does and in consequence the analytic projection of $g \in X^{\prime}$ belongs to $H X^{\prime}$.

In the final theorem of the paper we give a new characterization of belonging to the class $H X$ in terms of convolution with $L^{1}$ function.

Theorem 7. Let $X$ be an order continuous and maximal r.i. space on $\mathbb{T}$ and assume that $X$ possesses the Riesz property. For

$$
f=\sum_{n=0}^{\infty} \widehat{f}(n) u_{n} \in H(\mathbb{D})
$$

the following are equivalent:

(i) Function $f$ belongs to $H X$.

(ii) For any $g \in L^{1}(\mathbb{T})$ with the Fourier series

$$
\sum_{n=-\infty}^{\infty} \widehat{g}(n) e^{i n \theta}, \quad \theta \in \mathbb{T}
$$

the series

$$
\sum_{n=0}^{\infty} \widehat{f}(n) \hat{g}(n) u_{n}
$$

represents a function from space $H X$.

Proof. (i) $\Rightarrow$ (ii). Let us recall that if $f \in H^{p}, p \in[1, \infty)$, and $g \in L^{1}$, then $f^{*} * g \in L^{p}$ and by [13, Theorem 1.15] there exists a constant $C>0$ such that

$$
\left\|f^{*} * g\right\|_{L^{p}} \leqslant C\|f\|_{H^{p}} .
$$

It is easy to see that $\widehat{f^{*} * g}(n)=\widehat{f}(n) \widehat{g}(n)$ for $n \in \mathbb{N}_{0}$. Since $X$ has the Riesz property then there exist $p_{X}$ and $q_{X}$ such that $p_{X}, q_{X} \in(1, \infty)$. Thus two real numbers $p, q$ satisfying $1<p<p_{X} \leqslant q_{X}<q<\infty$ can be found. From Boyd's theorem (see [14]) it follows that $X$ is an interpolation space between $L^{p}$ and $L^{q}, p, q \in(1, \infty)$. Inequality (58) implies that the operator $T: H^{p} \rightarrow L^{p}$ given by $T f=f^{*} * g$ is bounded for any $p \in[1, \infty)$. By the interpolation property it follows that $T: H X \rightarrow X$ is well defined and bounded. Thus for any $f \in H X$ and $g \in L^{1}$ the convolution $f^{*} * g$ produces a function belonging to $X$. Since $X$ is maximal and thus $X^{\prime \prime}=X$ it follows that

$$
\sum_{n=0}^{\infty} \widehat{f}(n) \hat{g}(n) u_{n} \in H X
$$

(ii) $\Rightarrow$ (i). Observe that since

$$
\sum_{n=0}^{\infty} \widehat{f}(n) \widehat{g}(n) u_{n} \in H X
$$

for all $g \in L^{1}$ with Fourier coefficients $\{\widehat{g}(n)\}_{n=-\infty}^{\infty}$, then in particular the series

$$
\sum_{n=0}^{\infty} \widehat{f}(n) \hat{g}(-n) e^{-i n \theta}
$$

is a Fourier series of some $F \in X$. Let us define an operator $S: L^{1} / H_{0}^{1} \rightarrow X / X_{0}$ with the formula

$$
S\left(g+H_{0}^{1}\right)=F+H X_{0}, \quad g \in L^{1} .
$$

From the Closed Graph Theorem it follows that $S$ is a bounded operator since $f_{n} \rightarrow f$ in $L^{1} / H_{0}^{1}$ (resp., $X / X_{0}$ ) implies

$$
\lim _{n \rightarrow \infty} \widehat{f_{n}}(k)=\widehat{f}(k), \quad k \in \mathbb{N}_{0}
$$

Notice that the adjoint $S^{*}$ maps $\left(X / H X_{0}\right)^{*}$ into $\left(L^{1} / H_{0}^{1}\right)^{*}$ and, by Lemma 6, $S^{*}: \widetilde{H X^{\prime}} \rightarrow H^{\infty}$. From the definition of $S$ it follows that

$$
\int_{0}^{2 \pi} h^{*}\left(e^{i \theta}\right) F\left(e^{i \theta}\right) d \theta=\int_{0}^{2 \pi} S^{*} h\left(e^{i \theta}\right) g\left(e^{i \theta}\right) d \theta,
$$

$$
h \in H X^{\prime} \text {. }
$$

Now take $g\left(e^{i \theta}\right)=e^{i k \theta}, \theta \in \mathbb{T}, k \in \mathbb{N}_{0}$. The corresponding $F$ is given by $F\left(e^{i \theta}\right)=\widehat{f}(k) e^{-i k \theta}$ and by formula (64) it follows that $\widehat{S^{*} h}(k)=\widehat{f}(k) \widehat{h}(k)$. Thus $S^{*}$ is a Hadamard product of $f \in$ $H X$ and $h \in H X^{\prime}$ and from Theorem 4 we get $f \in H X$.

\section{Competing Interests}

The author declares that there is no conflict of interests regarding the publication of this paper.

\section{Acknowledgments}

The work was supported by The Foundation for Polish Science (FNP).

\section{References}

[1] J. Caveny, "Bounded Hadamard products of Hp functions," Duke Mathematical Journal, vol. 33, pp. 389-394, 1966.

[2] O. Blasco and M. Pavlović, "Coefficient multipliers on Banach spaces of analytic functions," Revista Matemática Iberoamericana, vol. 27, no. 2, pp. 415-447, 2011.

[3] P. Duren, Theory of Hp Spaces, Academic Press, San Diego, Calif, USA, 1976.

[4] R. A. Kortram, "A simple proof for Schur's theorem," Proceedings of the American Mathematical Society, vol. 129, no. 11, pp. 32113212, 2001. 
[5] M. Pavlović, "Analytic functions with decreasing coefficients and Hardy and Bloch spaces," Proceedings of the Edinburgh Mathematical Society, vol. 56, no. 2, pp. 623-635, 2013.

[6] S. M. Buckley, M. S. Ramanujan, and D. Vukotić, "Bounded and compact multipliers between Bergman and Hardy spaces," Integral Equations and Operator Theory, vol. 35, no. 1, pp. 1-19, 1999.

[7] J. Lindenstrauss and L. Tzafriri, Classical Banach Spaces, II, Function Spaces, Springer, Berlin, Germany, 1979.

[8] M. Mastyło and P. Mleczko, "Absolutely summing multipliers on abstract Hardy spaces," Acta Mathematica Sinica, vol. 25, no. 6, pp. 883-902, 2009.

[9] M. Mastyło and P. Mleczko, "Norm estimates for matrix operators between Banach spaces," Linear Algebra and Its Applications, vol. 438, no. 3, pp. 986-1001, 2013.

[10] M. Mastyło and L. Rodríguez-Piazza, "Carleson measures and embeddings of abstract Hardy spaces into function lattices," Journal of Functional Analysis, vol. 268, no. 4, pp. 902-928, 2015.

[11] F. Fehér, D. Gaşpar, and H. Johnen, "Normkonvergenz von Fourierreihen in rearrangement invarianten Banachräumen," Journal of Functional Analysis, vol. 13, no. 4, pp. 417-434, 1973.

[12] M. Lengfield, "A nested embedding theorem for Hardy-Lorentz spaces with applications to coefficient multiplier problems," Rocky Mountain Journal of Mathematics, vol. 38, no. 4, pp. 12151251, 2008.

[13] A. Zygmund, Trigonometric Series, I, Cambridge University Press, Cambridge, UK, 1959.

[14] D. W. Boyd, "Indices of function spaces and their relationship to interpolation," Canadian Journal of Mathematics, vol. 21, pp. 1245-1254, 1969. 


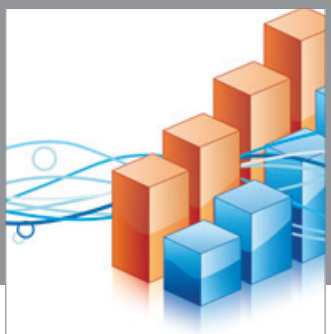

Advances in

Operations Research

vatem alat4

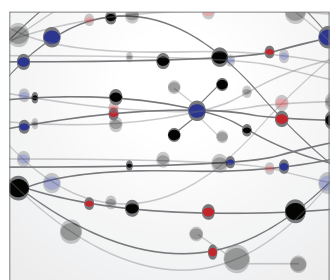

\section{The Scientific} World Journal
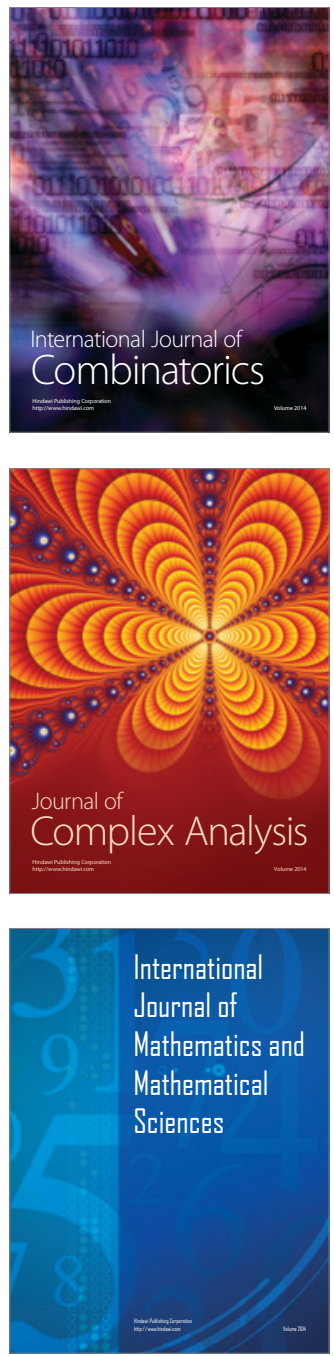
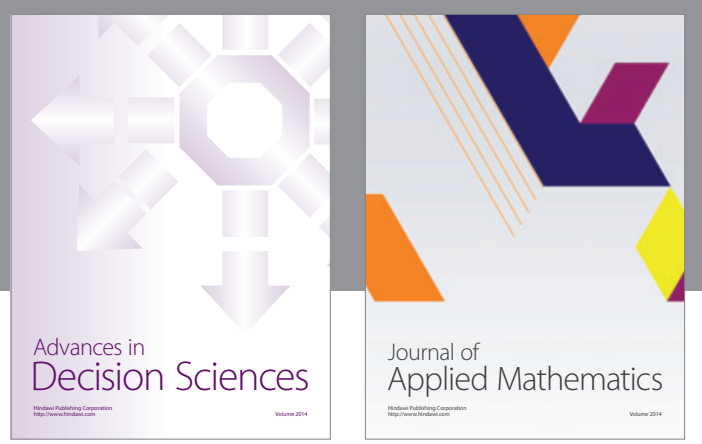

Algebra

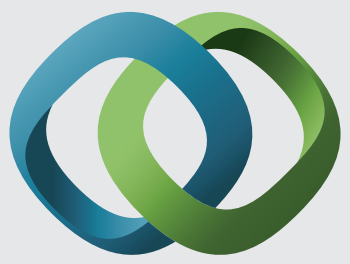

\section{Hindawi}

Submit your manuscripts at

http://www.hindawi.com
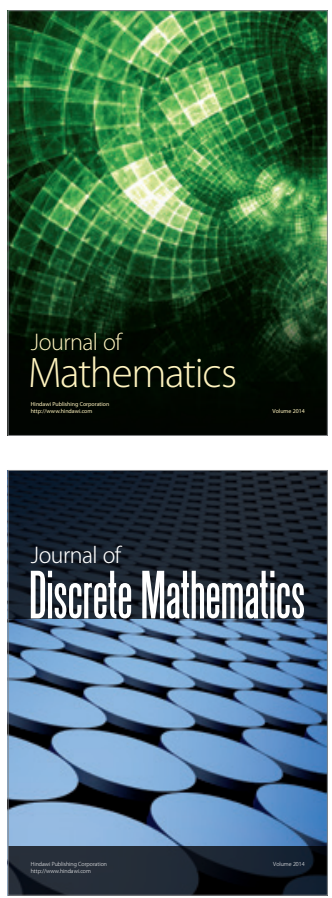

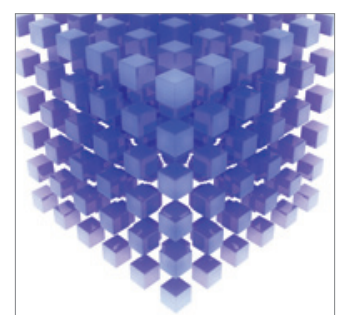

Mathematical Problems in Engineering
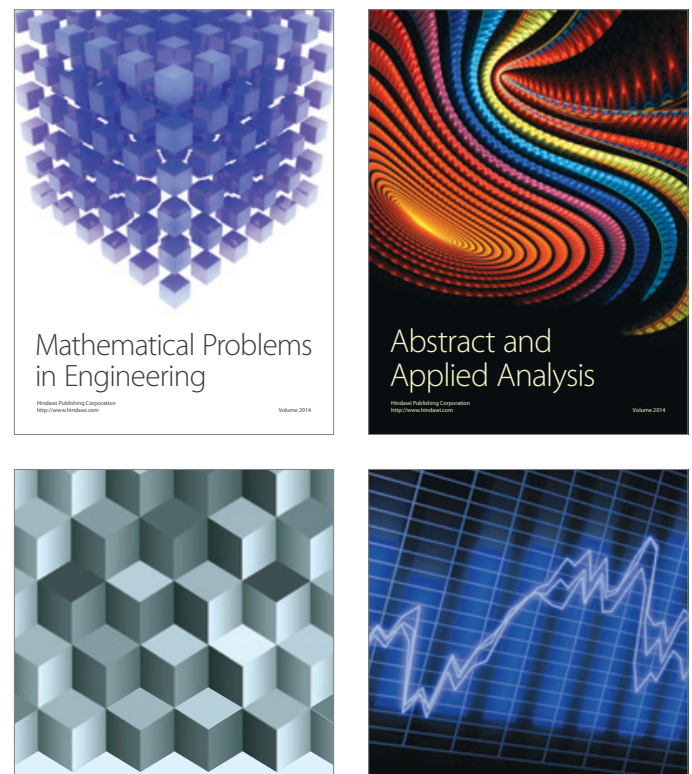

Journal of

Function Spaces

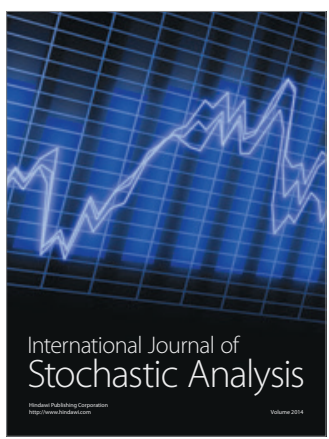

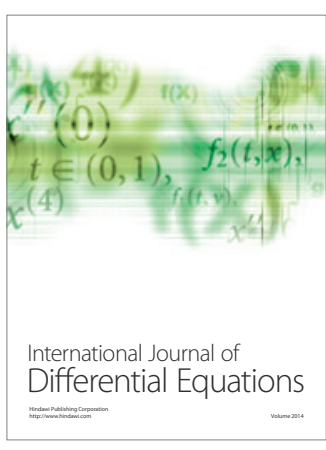
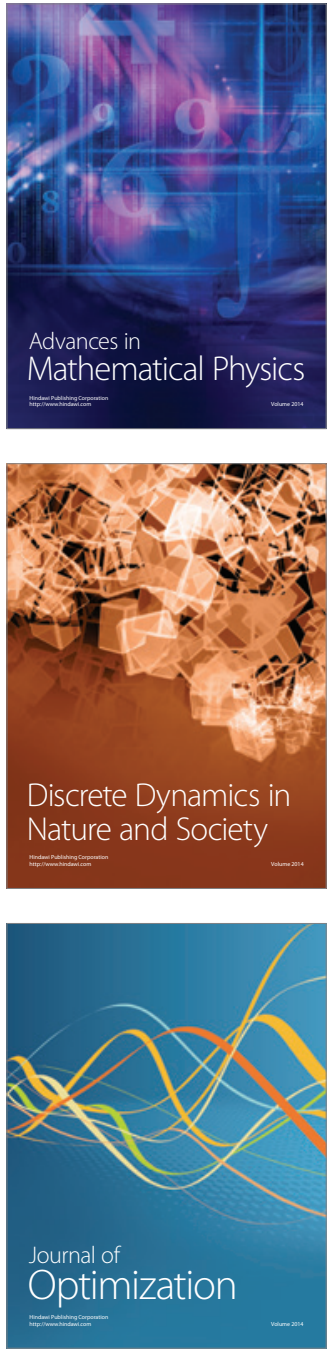